\title{
Kaluza-Klein Spectrometry for Supergravity
}

\author{
Emanuel Malek $\oplus^{1, *}$ and Henning Samtleben $\oplus^{2, \dagger}$ \\ ${ }^{1}$ Max-Planck-Institut für Gravitationsphysik (Albert-Einstein-Institut), Am Mühlenberg 1, 14476 Potsdam, Germany \\ ${ }^{2}$ Univ Lyon, Ens de Lyon, Univ Claude Bernard, CNRS, Laboratoire de Physique, F-69342 Lyon, France
}

(Received 9 December 2019; accepted 23 January 2020; published 10 March 2020)

\begin{abstract}
Exceptional field theories yield duality covariant formulations of supergravity. We show that they provide a highly efficient tool to compute the Kaluza-Klein mass spectra associated with compactifications around various background geometries relevant for string theory and holographic applications. This includes geometries with little to no remaining symmetries, hardly accessible to standard methods. As an illustration, we determine the masses of some higher Kaluza-Klein multiplets around warped geometries corresponding to some prominent $\mathcal{N}=2$ supersymmetric anti-de Sitter vacua in maximal supergravity.
\end{abstract}

DOI: $10.1103 /$ PhysRevLett.124.101601

An old and central problem in theories with extra dimensions is the determination of the mass spectrum of higher-dimensional fluctuations around a given compactification background. From a phenomenological point of view, this is central to the question of which particles are observable in lower dimensions. In particular, massless scalar fields in general contradict particle physics observations, while scalar fields of negative mass squared indicate a vacuum instability that jeopardizes the entire compactification scenario. This is particularly relevant for vacua whose stability is not controlled by supersymmetry arguments. In the holographic context, the full Kaluza-Klein spectrum around particular geometries with anti-de Sitter (AdS) factors carries vital information about the conformal dimensions of gauge invariant operators in the dual gauge theory.

Computation of Kaluza-Klein mass spectra, in general, is a highly nontrivial problem, which requires linearization and diagonalization of the higher-dimensional field equations expanded in terms of the eigenmodes of suitable Laplacian operators on the internal manifold. There are only particular scenarios where this problem has been fully solved. For manifolds with large isometry group and preserving major fractions of supersymmetry, fluctuations organize themselves into (semi)short multiplets of the superalgebra of background isometries, such that the internal harmonics are controlled by group theory, and the mass eigenvalues are essentially determined by the residual quantum numbers. This has underpinned the early work on the Kaluza-Klein spectra of the maximally supersymmetric backgrounds $\mathrm{AdS}_{4} \times S^{7}[1-4]$, and $\mathrm{AdS}_{5} \times S^{5}[5,6]$.

Published by the American Physical Society under the terms of the Creative Commons Attribution 4.0 International license. Further distribution of this work must maintain attribution to the author(s) and the published article's title, journal citation, and DOI. Funded by SCOAP ${ }^{3}$.
Examples of compactifications with less supersymmetry yet controlled by the coset structure of the internal spaces include Refs. [7,8].

For general manifolds, the problem is far more complicated. The mass spectrum of the spin- 2 sector shows some universal pattern and can be determined from a wave equation depending only on the background geometry and not on the supergravity matter fields [9]. This has been further exploited in [10-13]. By contrast, the fluctuation equations for the lower-spin fields, notably the scalar fluctuations, generically depend on the nonmetric details of the background solution, such as the nonvanishing background fluxes of $p$-forms. Moreover, these fluctuations mix together the various matter fields such that mass eigenstates have to be meticulously disentangled. This renders the general analysis highly nontrivial.

On supersymmetric backgrounds, the information from the spin-2 sector may be extrapolated to some of the other matter fields upon exploiting the multiplet structure of the fluctuations $[12,14,15]$. However, this approach offers only partial access to the Kaluza-Klein spectrum as it remains restricted within the spin-2 multiplets. Moreover, even for those mass eigenstates sitting inside spin-2 multiplets, the laborious task of identifying the corresponding fluctuations within the higher-dimensional theory remains. This is indispensable for any holographic application.

In this Letter, we will show that exceptional field theory [16] offers a very powerful tool to solve this problem for large classes of examples. Exceptional field theories yield a duality covariant formulation of higher-dimensional supergravity theories. They have proven instrumental in constructing consistent truncations from higher-dimensional supergravities $[17,18]$. In particular, they offer a constructive way to obtain the nonlinear reduction Ansätze of the higher-dimensional theory in terms of the fields of a lowerdimensional gauged supergravity, such that all solutions of the lower-dimensional theory induce solutions of the 
higher-dimensional field equations. In this Letter, we will demonstrate that this construction may be naturally extended to also produce the form of the higherdimensional fluctuations around any solution uplifted from the lower-dimensional theory. In particular, we find that the formalism automatically disentangles the higherdimensional fluctuation equations, which allows us to obtain compact and universal formulas for the mass matrices of the infinite Kaluza-Klein towers. We briefly illustrate the formalism for a couple of prominent $\mathcal{N}=2$ supersymmetric AdS vacua in maximal supergravity. Details will appear in an upcoming paper [19].

As an example, we discuss the domain solution of $D=5$ gauged supergravity that uplifts to a solution of type IIB supergravity interpolating between the maximally supersymmetric $\mathrm{AdS}_{5} \times S^{5}$ and a warped $\mathrm{AdS}_{5} \times M^{5}$ geometry [20]. The latter background is the conjectured holographic dual of the infrared (IR) fixed point of the renormalization group (RG) flow triggered by a mass deformation of $\mathcal{N}=4$ super-Yang-Mills theory. The internal manifold $M^{5}$ is a deformation of the round sphere $S^{5}$, preserving only $\mathrm{U}(2)$ isometries and breaking supersymmetry down to one quarter. Moreover, all $p$-forms in ten dimensions acquire nonvanishing background fluxes. Accordingly, this background is not amenable to standard techniques of harmonic analysis. We show that, within exceptional field theory, the full Kaluza-Klein spectrum around this background can be computed with the mass eigenstates neatly expressed in terms of the harmonics of the round $S^{5}$, and we give the explicit results for the first level. The same pattern applies to many other holographic backgrounds, including $[12,15,21-26]$ (to name a few) and will allow us to extract their hitherto unknown Kaluza-Klein spectra.

Let us start by briefly reviewing the structure of the relevant $E_{6(6)}$ exceptional field theory (ExFT); for details, we refer to [16,27]. This ExFT is a universal formulation of all higher-dimensional supergravities in terms of the fields of $D=5$ maximal supergravity. Its bosonic sector

$$
\begin{aligned}
\left\{g_{\mu \nu}, \mathcal{M}_{M N}, \mathcal{A}_{\mu}{ }^{M}, \mathcal{B}_{\mu \nu M}\right\}, \quad \mu & =0, \ldots, 4, \\
M & =1, \ldots, 27
\end{aligned}
$$

comprises an external and an internal metric $g_{\mu \nu}, \mathcal{M}_{M N}$, respectively, with the latter parametrizing the coset space $E_{6(6)} / \mathrm{USp}(8)$, together with vector and tensor fields $\mathcal{A}_{\mu}{ }^{M}$ and $\mathcal{B}_{\mu \nu M}$, transforming in the $\mathbf{2 7}$ and $\mathbf{2 7}$ of the group $E_{6(6)}$, respectively. All fields formally live on a $(5+27)-$ dimensional exceptional space-time with coordinates $\left\{x^{\mu}, Y^{M}\right\}$, subject to the section constraint

$$
d^{M N K} \partial_{N} \otimes \partial_{K}=0,
$$

with the symmetric $E_{6(6)}$ invariant $d$ symbol $d^{M N K}$. The ExFT Lagrangian resembles the generic structure of maximal supergravity in five dimensions,

$$
\begin{aligned}
\mathcal{L} \equiv & \hat{R}+\frac{1}{24} g^{\mu \nu} \mathcal{D}_{\mu} \mathcal{M}^{M N} \mathcal{D}_{\nu} \mathcal{M}_{M N} \\
& -\frac{1}{4} \mathcal{M}_{M N} \mathcal{F}^{\mu \nu M} \mathcal{F}_{\mu \nu}{ }^{N}+|g|^{-1 / 2} \mathcal{L}_{\text {top }}-V(\mathcal{M}, g) .
\end{aligned}
$$

Here, derivatives are covariantized $\mathcal{D}_{\mu}=\partial_{\mu}-\mathbb{L}_{A_{\mu}}$ with respect to generalized diffeomorphisms acting as

$\mathbb{L}_{\Lambda} \mathcal{M}_{M N}=\Lambda^{K} \partial_{K} \mathcal{M}_{M N}+12 \partial_{K} \Lambda^{L} \mathbb{P}^{K}{ }_{L}{ }^{P}{ }_{(M} \mathcal{M}_{N) P}$,

with the projector onto the adjoint representation

$$
\mathbb{P}^{M}{ }_{N}{ }^{K}{ }_{L}=\frac{1}{18} \delta_{N}^{M} \delta_{L}^{K}+\frac{1}{6} \delta_{N}^{K} \delta_{L}^{M}-\frac{5}{3} d_{N L R} d^{M K R} .
$$

The non-Abelian field strengths read

$\mathcal{F}_{\mu \nu}{ }^{M} \equiv 2 \partial_{[\mu} \mathcal{A}_{\nu]}{ }^{M}-\left[\mathcal{A}_{\mu}, \mathcal{A}_{\nu}\right]_{E}^{M}+10 d^{M N K} \partial_{K} \mathcal{B}_{\mu \nu N}$,

with the non-Abelian $E$ bracket [, $]_{E}$ derived from the action (4) and the coupling to 2 -forms $\mathcal{B}_{\mu \nu M}$ required in order to achieve gauge covariance. The topological term in (3) is obtained by integrating

$$
\begin{aligned}
d \mathcal{L}_{\text {top }} \propto & d_{M N K} \mathcal{F}^{M} \wedge \mathcal{F}^{N} \wedge \mathcal{F}^{K} \\
& -40 d^{M N K} \mathcal{H}_{M} \wedge \partial_{N} \mathcal{H}_{K},
\end{aligned}
$$

with $\mathcal{H}_{M}$ denoting the non-Abelian 3-form field strength of the tensor fields $\mathcal{B}_{M}$. Finally, the potential $V(\mathcal{M}, g)$ is a gauge invariant combination of terms bilinear in internal derivatives acting on internal and external metric.

The section constraint (2) implies that fields depend on no more than six of the internal coordinates. Upon inequivalent choices of the physical internal coordinates among the $\left\{Y^{M}\right\}$, the Lagrangian (3) reproduces that of the full $D=11$ and ten-dimensional IIB supergravity, respectively. Specifically, the IIB coordinates are identified upon breaking $E_{6(6)}$ down to $\mathrm{GL}(5) \times \mathrm{SL}(2)$ with

$$
\begin{aligned}
\left\{Y^{M}\right\} \rightarrow & \left\{Y^{m}, Y_{k m n}, Y_{m}{ }^{\alpha}, Y^{\alpha}\right\}, \\
& m=1, \ldots, 5 ; \quad \alpha=1,2,
\end{aligned}
$$

and restricting all field dependence to $\left\{x^{\mu}, Y^{m}\right\}$. Upon analogous decomposition of the ExFT fields (1) under $\mathrm{GL}(5) \times \mathrm{SL}(2)$, followed by proper on-shell dualizations and field redefinitions, one can establish the precise dictionary to recover the full field content of the tendimensional IIB theory [27].

ExFT has proven a powerful tool for the construction of consistent truncations. The reduction formulas for the ExFT fields (1) take the form of a generalized ScherkSchwarz Ansatz 


$$
\begin{aligned}
g_{\mu \nu}(x, Y) & =\rho^{-2}(Y) g_{\mu \nu}(x), \\
\mathcal{M}_{M N}(x, Y) & =U_{M} \underline{\underline{K}}(Y) U_{N} \underline{\underline{L}}(Y) M_{\underline{K L}}(x), \\
\mathcal{A}_{\mu}{ }^{M}(x, Y) & =\rho^{-1}(Y)\left(U^{-1}\right)_{\underline{N}}{ }^{M}(Y) A_{\mu} \underline{\underline{N}}(x), \\
\mathcal{B}_{\mu \nu M}(x, Y) & =\rho^{-2}(Y) U_{M} \underline{\underline{N}}(Y) B_{\mu \nu \underline{N}}(x),
\end{aligned}
$$

in terms of an $E_{6(6)}$ twist matrix $U$ and a weight factor $\rho$. The consistency conditions for the twist matrix are most compactly expressed as conditions of generalized Leibniz parallelizability [28]

$$
\mathbb{L}_{\mathcal{U}_{\underline{M}}} \mathcal{U}_{\underline{N}}=X_{\underline{M N}} \mathcal{U}_{\underline{K}}, \quad \text { for } \quad \mathcal{U}_{\underline{M}} \equiv \rho^{-1} U_{\underline{M}}^{-1},
$$

with constant embedding tensor $X_{M N}$. Once the twist matrix satisfies the consistency conditions (10), all dependence on the internal coordinates factors out from the IIB equations of motion, which then reduce to the equations of motion of maximal $D=5$ supergravity with gauging defined by the embedding tensor (10).

The $S^{5}$ reduction of IIB supergravity is described by a particular twist matrix living in $\mathrm{SL}(6) \subset E_{6(6)}$, induced by the $6 \times 6$ matrix

$$
\begin{aligned}
\left(U^{-1}\right)_{A}^{\hat{m}} & =\left\{\left(U^{-1}\right)_{A}{ }^{0},\left(U^{-1}\right)_{A}{ }^{m}\right\} \\
& =\stackrel{\circ}{\omega}^{1 / 3}\left\{\stackrel{\circ}{\omega}^{-1} \mathcal{Y}^{A}, \stackrel{\circ}{g}^{m n} \partial_{n} \mathcal{Y}^{A}+4 \check{\zeta}^{m} \mathcal{Y}^{A}\right\},
\end{aligned}
$$

in terms of elementary sphere harmonics $\mathcal{Y}^{A} \mathcal{Y}^{A}=1$ $(A=1, \ldots, 6)$, the round $S^{5}$ metric $\stackrel{\circ}{g}_{m n}=\partial_{m} \mathcal{Y}^{A} \partial_{n} \mathcal{Y}^{A}$, and the vector field $\stackrel{o}{\zeta}^{n}$ defined by $\stackrel{\circ}{\nabla}_{n} \zeta^{n}=1$. The weight factor is given by $\rho=\stackrel{\circ}{\omega}^{-1 / 3}$ in terms of the metric determinant $\stackrel{\circ}{\omega}^{2}=\operatorname{det} \stackrel{\circ}{g}_{m n}$. With the embedding of $\mathrm{SL}(6) \times \mathrm{SL}(2) \subset E_{6(6)}$ described by the breaking of the 27 as

$$
A^{\underline{M}} \rightarrow\left\{A^{A B}, A_{A \alpha}\right\}=\left\{A^{[A B]}, A_{A \alpha}\right\},
$$

the induced $E_{6(6)}$ twist matrix $\left(\mathcal{U}_{\left(S^{5}\right)}\right)_{\underline{M}}{ }^{M}$ satisfies (10) with the nonzero components of the embedding tensor given by

$$
X_{\underline{M N}}^{\underline{K}}:\left\{\begin{array}{l}
X_{A B, C D}{ }^{E F}=2 \sqrt{2} \delta_{[A}^{[E} \delta_{B][C} \delta_{D]}^{F]}, \\
X_{A B}{ }^{C \alpha}{ }_{D \beta}=-\sqrt{2} \delta_{[A}^{C} \delta_{B] D} \delta_{\beta}^{\alpha} .
\end{array}\right.
$$

In particular, the twist matrix satisfies the relation

$$
\mathcal{U}_{\underline{M}}^{N} \partial_{N}=\mathcal{K}_{\underline{M}}^{m} \partial_{m}
$$

with the SO(6) Killing vector fields

$$
\mathcal{K}_{A B}{ }^{m}=\sqrt{2}^{\circ}{ }^{m n} \mathcal{Y}^{[A} \partial_{n} \mathcal{Y}^{B]}, \quad \mathcal{K}^{A \alpha m}=0 .
$$

Within the reduction Ansatz (9), the $\mathrm{AdS}_{5} \times S^{5}$ solution of IIB supergravity takes the simple form

$$
g_{\mu \nu}(x)=\left(g_{\mathrm{AdS}_{5}}\right)_{\mu \nu}(x), \quad M_{\underline{M N}}(x)=\delta_{\underline{M N}},
$$

with vectors and tensors vanishing. Fluctuations of the IIB theory around this background solution organize into an infinite tower of short Kaluza-Klein multiplets of increasing masses [5,6]. The Ansatz (9) describes the full nonlinear embedding into ten dimensions of the lowest (massless) Kaluza-Klein multiplet that carries the field content of $D=5$ maximal gauged supergravity [29], such that every solution of the $D=5$ theory lifts to a solution of the IIB field equations [30].

In this Letter, we address the higher Kaluza-Klein multiplets. In the standard formulation of IIB supergravity, fluctuations are formulated in terms of appropriate sphere harmonics. For example, a ten-dimensional scalar field gives rise to a tower of $D=5$ scalar fields

$$
\phi(x, Y)=\sum_{\Sigma} \mathcal{Y}^{\Sigma} \varphi_{\Sigma}(x),
$$

accompanying the scalar harmonics $\mathcal{Y}^{\Sigma}$ on the round $S^{5}$, i.e., the sphere functions on which the Killing vector fields (15) have a linear action

$$
\mathcal{K}_{\underline{M}}{ }^{m} \partial_{m} \mathcal{Y}^{\Sigma}=-\mathcal{T}_{\underline{M}}{ }^{\Sigma \Omega} \mathcal{Y}^{\Omega},
$$

with $\mathrm{SO}(6)$ generators $\mathcal{T}_{\underline{M}}$. Specifically, for $S^{5}$ these harmonics can be expressed as polynomials in the elementary harmonics $\mathcal{Y}^{A}$ as

$$
\left\{\mathcal{Y}^{\Sigma}\right\}=\left\{1, \mathcal{Y}^{A}, \mathcal{Y}^{A_{1} A_{2}}, \ldots, \mathcal{Y}^{A_{1} \ldots A_{n}}, \ldots\right\}
$$

where we denote by $\left.\mathcal{Y}^{A_{1} \ldots A_{n}} \equiv \mathcal{Y}^{\left(\left(A_{1}\right.\right.} \ldots \mathcal{Y}^{\left.A_{n}\right)}\right)$ traceless symmetrization. The index $\Sigma$ thus runs over the tower of symmetric vector representations $[n, 0,0]$ of $\mathrm{SO}(6)$. For the fields of nonvanishing spin, the relevant harmonics on coset spaces such as $S^{5}=\mathrm{SO}(6) / \mathrm{SO}(5)$ can be classified and determined by group theoretical methods [31].

The main result that we will exploit in this Letter is the observation that, in terms of the ExFT variables (1), fluctuations around the background (16) are most compactly expressed by combining the nonlinear embedding of the lowest multiplet (9) with the infinite tower of scalar harmonics $\mathcal{Y}^{\Sigma}$. More precisely, for vector and tensor fields, the full set of IIB fluctuations is described by the generalization of (9) to

$$
\begin{aligned}
& \mathcal{A}_{\mu}{ }^{M}=\rho^{-1}\left(U^{-1}\right)_{\underline{N}}{ }^{M} \sum_{\Sigma} \mathcal{Y}^{\Sigma} A_{\mu}{ }^{\underline{N}, \Sigma}(x), \\
& \mathcal{B}_{\mu \nu M}=\rho^{-2} U_{M^{\underline{N}}} \sum_{\Sigma} \mathcal{Y}^{\Sigma} B_{\mu \nu \underline{N}, \Sigma}(x),
\end{aligned}
$$

with the sum running over scalar harmonics (19). For the external and the internal metric, the Ansatz 


$$
\begin{aligned}
g_{\mu \nu} & =\rho^{-2}\left(\left(g_{\mathrm{AdS}_{5}}\right)_{\mu \nu}(x)+\sum_{\Sigma} \mathcal{Y}^{\Sigma} h_{\mu \nu, \Sigma}(x)\right), \\
\mathcal{M}_{M N} & =U_{M^{\underline{K}}} U_{N} \underline{\underline{L}}\left(\delta_{\underline{K L}}+\sum_{\Sigma} \mathcal{Y}^{\Sigma} j_{\underline{K L}, \Sigma}(x)\right),
\end{aligned}
$$

is given in terms of fluctuations, further restricted by the fact that these metrics parametrize the coset spaces $\mathrm{GL}(5) / \mathrm{SO}(5)$ and $E_{6(6)} / \mathrm{USp}(8)$, respectively.

The conditions (10) satisfied by the twist matrices ensure that, with this Ansatz to linear order in the fluctuations, all dependence on the internal coordinates still factors out from the equations of motion. The latter thus reduce to linear five-dimensional differential equations. In particular, in the IIB field equations, internal derivatives act throughout via the combination of (10) and (14); i.e., their action on the sphere harmonics is realized by the action (18) of Killing vector fields. Consequently, the resulting equations do not mix fluctuations over different $\mathrm{SO}(6)$ representations $\Sigma$. The same structure underlies the ExFT supersymmetry transformations [32]. As a result, all fluctuations in (20) and (21) associated with a fixed $\mathrm{SO}(6)$ representation $\Sigma=[n, 0,0]$ combine into a single $1 / 2$-BPS multiplet $\operatorname{BPS}[n]$. This is to be contrasted with the structure in the original IIB variables: after evaluating the products of the sphere harmonics $\mathcal{Y}^{\Sigma}$ with the $Y$-dependent twist matrices in (20) and (21) and translating the ExFT fields back into the IIB supergravity fields, fluctuations of the original IIB fields combine linear combinations of different mass eigenstates originating from different BPS multiplets.

We now compute the mass matrices by plugging the Ansatz (20) and (21) into the equations of motion. For the tensor fields, the Lagrangian (3) gives rise to the first-order duality equations

$$
d^{P M L} \partial_{L}\left(\mathcal{M}_{M N} \mathcal{F}^{\mu \nu N}+\kappa \epsilon^{\mu \nu \rho \sigma \tau} \mathcal{H}_{\rho \sigma \tau M}\right)=0,
$$

with $\kappa^{2} \equiv \frac{5}{32}$. With the field strengths (6) carrying a Stuickelberg-type coupling to the 2-forms, linearization and gauge fixing of (22), together with an evaluation of internal derivatives on twist matrices and scalar harmonics, gives rise to fluctuation equations for topologically massive 2 -forms

$$
3 \partial_{[\mu} B_{\nu \rho] \underline{M} \alpha}=\frac{1}{2} \varepsilon_{\mu \nu \rho \sigma \tau} M \underline{M \Sigma} \underline{\underline{N} \Omega} B_{\underline{N \alpha}}^{\sigma \tau},
$$

with the antisymmetric mass matrix given by

$$
M \underline{M} \Sigma, \underline{N} \Omega \propto 2 d \underline{M K L} X_{\underline{K L}^{\underline{N}}} \delta^{\Sigma \Omega}-10 d \underline{\underline{M N K}}^{\mathcal{T}_{\underline{K}}}{ }^{\Sigma \Omega} .
$$

It exhibits a very intriguing form as a superposition of the mass matrix of the $D=5$ supergravity describing the lowest Kaluza-Klein multiplet with the SO(6) action (18) on the scalar harmonics.
A similar, although more lengthy computation, linearizing the second-order vector field equations descending from (3) yields the vector mass operator

$$
\begin{aligned}
M_{\underline{M} \Sigma, \underline{N} \Omega} \propto & \frac{1}{3} X_{\underline{M L}}^{\mathrm{s}} \underline{K}_{\underline{N K}}^{\mathrm{s}} \underline{\underline{L}} \delta^{\Sigma \Omega} \\
& +2\left(X_{\underline{M K}}^{\mathrm{s}}-X_{\underline{N M}}^{\mathrm{s}}\right) \mathcal{T}_{\underline{K}, \Omega \Sigma} \\
& -6\left(\mathbb{P} \underline{\underline{K}}_{\underline{M}}^{\underline{\underline{L}}}{ }_{\underline{N}}+\mathbb{P} \underline{\underline{M}}_{\underline{K}} \underline{\underline{L}}{ }_{\underline{N}}\right) \mathcal{T}_{\underline{L}, \Omega \Lambda} \mathcal{T}_{\underline{K}, \Lambda \Sigma} \\
& +\frac{8}{3} \mathcal{T}_{\underline{N}, \Omega \Lambda} \mathcal{T}_{\underline{M}, \Lambda \Sigma},
\end{aligned}
$$

in terms of the symmetrized $X_{\underline{M N}}^{\mathrm{s}} \equiv X_{\underline{M N}}^{\underline{K}}+X_{\underline{M K}}^{\underline{\underline{N}}}$ and the adjoint projector (5). Again, this formula combines the mass matrix of the $D=5$ supergravity with the $\mathrm{SO}(6)$ action (18). Finally, a similar formula can be derived for the scalar fluctuations (21). For the spin-2 fluctuations, the Ansatz (21) yields the simple mass formula $M_{\Sigma, \Omega} \propto$ $\mathcal{T}_{\underline{M}, \Sigma \Lambda} \mathcal{T}_{\underline{M}, \Lambda \Omega}$, coinciding with $[9,13]$.

In order to diagonalize the mass matrices level by level in the harmonics (19), we need to evaluate the formulas (24) and (25) with the explicit form of the embedding tensor (13), as well as the explicit expressions for the $E_{6(6)}$ tensor $d^{K M N}$ and the $\mathrm{SO}(6)$ action (18) in the basis (12)

$$
\begin{aligned}
d^{A B, C D, E F} & =\frac{1}{\sqrt{80}} \varepsilon^{A B C D E F}, \quad d^{A B}{ }_{C \alpha, D \beta}=\frac{1}{\sqrt{5}} \delta_{C D}^{A B} \varepsilon_{\alpha \beta}, \\
\left(\mathcal{T}_{A B}\right)_{C D} & =\sqrt{2} \delta_{C[A} \delta_{B] D} .
\end{aligned}
$$

It is then a straightforward exercise to determine the mass eigenvalues of the different $\mathrm{SO}(6)$ representations at level $\Sigma=[n, 0,0]$, i.e., of the different irreducible representations of $A^{A B, C_{1} \ldots C_{n}}, A_{A \alpha} C_{1} \ldots C_{n}$, etc. We summarize the result in Table I, which agrees with $[5,6]$. Moreover, it confirms that the propagating fluctuations described by the Ansatz (20) and (21) at level $\Sigma=[n, 0,0]$ precisely span the bosonic part of a single 1/2-BPS multiplet. Moreover, the Ansatz together with the dictionary of ExFT into IIB supergravity allows us to directly localize the different components of the BPS multiplets within the IIB theory.

While this allows for a very compact rederivation of the known results for $S^{5}$, the construction has vastly more farreaching applications. Since the reduction Ansatz is exact to

TABLE I. Mass spectrum (24) and (25) on round $S^{5}$ at level $n$.

\begin{tabular}{lcc}
\hline \hline Fluctuation & $\mathrm{SO}(6)$ & $m^{2}$ \\
\hline$A_{\mu}{ }^{A\left(\left(B, C_{1} C_{2} \ldots C_{n}\right)\right)}$ & {$[n, 1,1]$} & $n(n+2)$ \\
$A_{\mu}{ }^{B\left[C_{1}, C_{2}\right] C_{3} \ldots C_{n} B}$ & {$[n-2,1,1]$} & $(n+2)(n+4)$ \\
$A_{\mu}{ }^{\alpha\left[A, C_{1}\right] C_{2} \ldots C_{n}}$ & {$[n-1,1,1]$} & $(n+1)(n+3)$ \\
$B_{\mu \nu}{ }^{\alpha\left(\left(A, C_{1} \ldots C_{n}\right)\right)}$ & {$[n+1,0,0]$} & $(n+1)^{2}$ \\
$B_{\mu \nu}{ }^{\alpha B, B C_{2} \ldots C_{n}}$ & {$[n-1,0,0]$} & $(n+3)^{2}$ \\
$B_{\mu \nu}{ }^{\left[A B, C_{1}\right] C_{2} \ldots C_{n}}$ & {$[n-1,0,2]+$ c.c. } & $(n+2)^{2}$ \\
\hline \hline
\end{tabular}


all orders in the lowest multiplet, we may export the formulas to derive the Kaluza-Klein spectrum around any solution of the $D=5$ supergravity.

As an example, we consider the $\mathcal{N}=2$ supersymmetric stationary point in the $D=5$ scalar potential conjectured to be dual to the IR fixed point of the RG flow triggered by a mass deformation of maximal super-Yang-Mills theory [20]. It is represented by a twist matrix

$$
\mathcal{U}_{\underline{M}}{ }^{M}=\mathcal{V}_{\underline{M}}{ }^{N}\left(\mathcal{U}_{S^{5}}\right)_{\underline{N}}{ }^{M},
$$

where the constant $E_{6(6)}$ matrix $\mathcal{V}_{M^{-}}$identifies the location of the stationary point on the coset manifold $E_{6(6)} / \mathrm{SU}(8)$. Accordingly, the fluctuation Ansatz (20) and (21) holds with the new twist matrix (27), and the mass formulas (24) and (25) hold with embedding tensor $X_{\underline{M N}} \underline{K}$ and $\mathrm{SO}(6)$

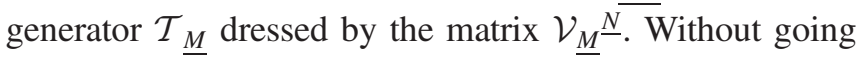
into details, we give the resulting mass spectrum around this vacuum at level 1 organized into multiplets of $\mathrm{SU}(2) \times$ $\mathrm{SU}(2,2 \mid 1)$ (in the notation of [20])

$$
\begin{array}{rl}
\text { 0: } D & \left(1+\frac{1}{2} \sqrt{37}, 0,0 ; 1\right)_{\mathbb{C}}+D\left(1+\frac{1}{2} \sqrt{61}, 0,0 ; 1\right)_{\mathbb{C}} \\
& +D_{S}\left(\frac{9}{2}, \frac{1}{2}, \frac{1}{2} ; 1\right)_{\mathbb{C}}+2 D_{S}\left(\frac{9}{2}, \frac{1}{2}, 0 ;-1\right)_{\mathbb{C}} \\
& +D\left(\frac{9}{2}, \frac{1}{2}, 0 ; 1\right)_{\mathbb{C}}, \\
\frac{\mathbf{1}}{\mathbf{2}}: D & \left(1+\frac{1}{4} \sqrt{145}, \frac{1}{2}, \frac{1}{2} ; \frac{1}{2}\right)_{\mathbb{C}}+D\left(1+\frac{1}{4} \sqrt{193}, 0,0 ; \frac{1}{2}\right)_{\mathbb{C}} \\
& +D\left(\frac{15}{4}, \frac{1}{2}, 0 ; \frac{1}{2}\right)_{\mathbb{C}}+D\left(\frac{17}{4}, \frac{1}{2}, 0 ;-\frac{1}{2}\right)_{\mathbb{C}} \\
& +D_{S}\left(\frac{15}{4}, 0,0 ; \frac{5}{2}\right)_{\mathbb{C}}+D_{S}\left(\frac{17}{4}, 0,0 ; \frac{3}{2}\right)_{\mathbb{C}}, \\
\mathbf{1}: 2 & D(1+\sqrt{7}, 0,0 ; 0)+D\left(1+\sqrt{7}, \frac{1}{2}, 0 ; 0\right)_{\mathbb{C}} \\
& +D_{S}\left(\frac{7}{2}, \frac{1}{2}, 0 ; 1\right)_{\mathbb{C}}+D_{S}\left(3, \frac{1}{2}, 0 ; 2\right)_{\mathbb{C}} \\
\frac{\mathbf{3}}{\mathbf{2}} D_{S} & \left(\frac{9}{4}, 0,0 ; \frac{3}{2}\right) \mathbb{C}_{\mathbb{C}}
\end{array}
$$

it is a nontrivial consistency check that the masses obtained by our formulas consistently combine into $\mathrm{SU}(2,2 \mid 1)$ multiplets. By $D_{S}$ we denote semishort multiplets whose energy saturates one of the unitarity bounds [33]. In contrast, the energy of the long multiplets cannot be deduced from representational arguments, but only from direct computation as presented here. Complex multiplets $D\left(E_{0}, j_{1}, j_{2} ; r\right)_{\mathbb{C}}$ come in pairs with their conjugates $D\left(E_{0}, j_{2}, j_{1} ;-r\right)_{\mathbb{C}}$. It is interesting to note that the complex multiplet $D_{S}\left(\frac{7}{2}, \frac{1}{2}, 0 ; 1\right)_{\mathbb{C}}$ contains two massless scalars.
As a second example, we study the $\mathrm{U}(3)$ invariant $\mathcal{N}=2$ $\mathrm{AdS}_{4}$ vacuum identified in the scalar potential of maximal $D=4$ gauged supergravity [34,35], conjectured to be the holographic dual of certain matter-coupled ChernSimons theories. The general multiplet structure of the Kaluza-Klein spectrum has been analyzed in [14] by group theoretical methods, which however do not give access to the masses of the long multiplets. Adapting the above mass formula (25) to $E_{7(7)}$ ExFT [36] allows us to straightforwardly determine the full spectrum. We list our result for the energies of the long $\mathrm{OSp}(2 \mid 4) \times \mathrm{SU}(3)$ multiplets appearing at the first level (in the notation of [14])

$$
\begin{aligned}
& \text { LVEC: }[0,0]: E_{0}=\frac{1}{2}+\frac{1}{2} \sqrt{33}, \\
& \text { LGRAV: }[1,0]+[0,1]: E_{0}=\frac{1}{2}+\frac{1}{6} \sqrt{145}, \\
& \text { LGINO: }[1,0]+[0,1]: E_{0}=\frac{17}{6}, \\
& \text { LVEC: }[1,0]+[0,1]: E_{0}=\frac{1}{2}+\frac{1}{6} \sqrt{217}, \\
& \text { LVEC: }[2,0]+[0,2]: E_{0}=\frac{7}{3}, \\
& \text { LGINO: }[1,1]: E_{0}=\frac{1}{2}+\sqrt{3}
\end{aligned}
$$

This extends the result of [15] for the long graviton multiplet (LGRAV) to all the long multiplets (the long vector, LVEC, and long gravitino, LGINO, multiplets) at this level. In particular, the energy values we find establish that there is no multiplet shortening occurring for these multiplets.

Our mass formulas (24) and (25) thus offer direct access to the full Kaluza-Klein spectra around these squashed and stretched spheres, hardly accessible to standard methods. As another intriguing application, the Ansatz (20) and (21), being exact in the lowest Kaluza-Klein multiplet, will allow us to compute within ExFT the holographic 2-point correlation functions of arbitrary operators throughout the renormalization group flows [20,37-39] described as domain wall solutions of lower-dimensional supergravities.

Further applications include similar analyses for the AdS vacua identified and studied in [12,15,21-26]. More generally, it will be interesting to combine the presented technology with recent numerical advances in searching for such vacua [40]. Of special interest are the nonsupersymmetric AdS vacua whose stability so far could only have been addressed within the lowest Kaluza-Klein multiplet. The technology presented here gives direct access to their notoriously difficult stability analysis. This is particularly interesting in light of recent conjectures by Ooguri and Vafa on the absence of such vacua [41]. Another interesting direction is the generalization of this framework to vacua within consistent truncations preserving smaller fractions of supersymmetry [18], giving access to yet larger classes of relevant $\mathrm{AdS}$ vacua. 
We would like to acknowledge the Mainz Institute for Theoretical Physics (MITP) of the Cluster of Excellence PRISMA+ (Project ID 39083149) for hospitality while this work was initiated. E. M. is supported by the ERC Advanced Grant "Exceptional Quantum Gravity" (Grant No. 740209).

*emanuel.malek@aei.mpg.de

†henning.samtleben@ens-lyon.fr

[1] F. Englert and H. Nicolai, in Group Theoretical Methods in Physics: Proceedings, 12th International Colloquium, Trieste, Italy, 1983 (Springer, Berlin, Heidelberg, 1983), pp. 249-283.

[2] E. Sezgin, Phys. Lett. 138B, 57 (1984).

[3] B. Biran, A. Casher, F. Englert, M. Rooman, and P. Spindel, Phys. Lett. 134B, 179 (1984).

[4] A. Casher, F. Englert, H. Nicolai, and M. Rooman, Nucl. Phys. B243, 173 (1984).

[5] M. Günaydin and N. Marcus, Classical Quantum Gravity 2, L11 (1985).

[6] H. J. Kim, L. J. Romans, and P. van Nieuwenhuizen, Phys. Rev. D 32, 389 (1985).

[7] A. Ceresole, G. Dall'Agata, R. D'Auria, and S. Ferrara, Phys. Rev. D 61, 066001 (2000).

[8] P. Fré, L. Gualtieri, and P. Termonia, Phys. Lett. B 471, 27 (1999).

[9] C. Bachas and J. Estes, J. High Energy Phys. 06 (2011) 005.

[10] A. Passias and A. Tomasiello, J. High Energy Phys. 12 (2016) 050.

[11] Y. Pang, J. Rong, and O. Varela, J. High Energy Phys. 01 (2018) 061.

[12] M. Gutperle, C. F. Uhlemann, and O. Varela, J. High Energy Phys. 07 (2018) 091.

[13] K. Dimmitt, G. Larios, P. Ntokos, and O. Varela, arXiv:1911.12202.

[14] I. Klebanov, T. Klose, and A. Murugan, J. High Energy Phys. 03 (2009) 140.

[15] I. R. Klebanov, S. S. Pufu, and F. D. Rocha, J. High Energy Phys. 06 (2009) 019.

[16] O. Hohm and H. Samtleben, Phys. Rev. Lett. 111, 231601 (2013).

[17] O. Hohm and H. Samtleben, J. High Energy Phys. 01 (2015) 131.
[18] E. Malek, Fortschr. Phys. 65, 1700061 (2017).

[19] E. Malek and H. Samtleben (to be published).

[20] D. Z. Freedman, S. S. Gubser, K. Pilch, and N. P. Warner, Adv. Theor. Math. Phys. 3, 363 (1999).

[21] E. D'Hoker, J. Estes, and M. Gutperle, J. High Energy Phys. 06 (2007) 021.

[22] T. Fischbacher, K. Pilch, and N.P. Warner, arXiv: 1010.4910.

[23] N. Bobev, K. Pilch, and N. P. Warner, J. High Energy Phys. 06 (2014) 058.

[24] H. Godazgar, M. Godazgar, O. Krüger, H. Nicolai, and K. Pilch, J. High Energy Phys. 01 (2015) 056.

[25] A. Guarino, D. L. Jafferis, and O. Varela, Phys. Rev. Lett. 115, 091601 (2015).

[26] N. Bobev, T. Fischbacher, and K. Pilch, J. High Energy Phys. 01 (2020) 099.

[27] A. Baguet, O. Hohm, and H. Samtleben, Proc. Sci., CORFU2014 (2015) 133.

[28] K. Lee, C. Strickland-Constable, and D. Waldram, Fortschr. Phys. 65, 1700048 (2017).

[29] M. Günaydin, L. J. Romans, and N. P. Warner, Nucl. Phys. B272, 598 (1986).

[30] A. Baguet, O. Hohm, and H. Samtleben, Phys. Rev. D 92 , 065004 (2015).

[31] A. Salam and J. A. Strathdee, Ann. Phys. (N.Y.) 141, 316 (1982).

[32] E. Musaev and H. Samtleben, J. High Energy Phys. 03 (2015) 027.

[33] M. Flato and C. Fronsdal, Lett. Math. Phys. 8, 159 (1984).

[34] B. de Wit and H. Nicolai, Nucl. Phys. B208, 323 (1982).

[35] N. P. Warner, Phys. Lett. 128B, 169 (1983).

[36] O. Hohm and H. Samtleben, Phys. Rev. D 89, 066017 (2014).

[37] R. Corrado, K. Pilch, and N. P. Warner, Nucl. Phys. B629, 74 (2002).

[38] M. Bianchi, O. DeWolfe, D. Z. Freedman, and K. Pilch, J. High Energy Phys. 01 (2001) 021.

[39] M. Bianchi, D. Z. Freedman, and K. Skenderis, J. High Energy Phys. 08 (2001) 041.

[40] I. M. Comsa, M. Firsching, and T. Fischbacher, J. High Energy Phys. 08 (2019) 057.

[41] H. Ooguri and C. Vafa, Adv. Theor. Math. Phys. 21, 1787 (2017). 